\title{
The Effect of Gadolinium on the ESR Response of Alanine and Ammonium Tartrate Exposed to Thermal Neutrons
}

\author{
Author(s): Maurizio Marrale, Maria Brai, Gaetano Gennaro, Antonio Bartolotta, and Maria Cristina
} D'Oca

Source: Radiation Research, 169(2):232-239.

Published By: Radiation Research Society

DOI: http://dx.doi.org/10.1667/RR1138.1

URL: http://www.bioone.org/doi/full/10.1667/RR1138.1

BioOne (www.bioone.org) is a nonprofit, online aggregation of core research in the biological, ecological, and environmental sciences. BioOne provides a sustainable online platform for over 170 journals and books published by nonprofit societies, associations, museums, institutions, and presses.

Your use of this PDF, the BioOne Web site, and all posted and associated content indicates your acceptance of BioOne's Terms of Use, available at www.bioone.org/page/terms_of_use.

Usage of BioOne content is strictly limited to personal, educational, and non-commercial use. Commercial inquiries or rights and permissions requests should be directed to the individual publisher as copyright holder. 


\title{
The Effect of Gadolinium on the ESR Response of Alanine and Ammonium Tartrate Exposed to Thermal Neutrons
}

\author{
Maurizio Marrale ${ }^{a, c, 1}$ Maria Brai, ${ }^{a, c}$ Gaetano Gennaro, ${ }^{a}$ Antonio Bartolotta ${ }^{b, c}$ and Maria Cristina D’Oca ${ }^{b}$ \\ ${ }^{a}$ Dipartimento di Fisica e Tecnologie Relative, Università di Palermo, Viale delle Scienze, 90128 Palermo, Italy; ${ }^{b}$ Dipartimento Farmacochimico \\ Tossicologico e Biologico, Università di Palermo, 90123 Palermo, Italy; and c Unità CNISM, Palermo and Gruppo V, Sezione INFN, Catania, Italy
}

Marrale, M., Brai, M., Gennaro, G., Bartolotta, A. and D'Oca, M. C. The Effect of Gadolinium on the ESR Response of Alanine and Ammonium Tartrate Exposed to Thermal Neutrons. Radiat. Res. 169, 232-239 (2008).

Many efforts have been made to develop neutron capture therapy (NCT) for cancer treatment. Among the challenges in using NCT is the characterization of the features of the mixed radiation field and of its components. In this study, we examined the enhancement of the ESR response of pellets of alanine and ammonium tartrate with gadolinium oxide exposed to a thermal neutron beam. In particular, the ESR response of these dosimeters as a function of the gadolinium content inside the dosimeter was analyzed. We found that the addition of gadolinium improves the sensitivity of both alanine and ammonium tartrate. However, the use of gadolinium reduces or abolishes tissue equivalence because of its high atomic number $\left(Z_{\mathrm{Gd}}=64\right)$. Therefore, it is necessary to find the optimum compromise between the sensitivity to thermal neutrons and the reduction of tissue equivalence. Our analysis showed that a low concentration of gadolinium oxide (of the order of $5 \%$ of the total mass of the dosimeter) can enhance the thermal neutron sensitivity more than 13 times with an insignificant reduction of tissue equivalence. $\odot 2008$ by Radiation Research Society

\section{INTRODUCTION}

Neutron capture therapy (NCT) is an experimental cancer treatment based on the administration of suitable compounds, usually containing ${ }^{10} \mathrm{~B}$ or ${ }^{157} \mathrm{Gd}$, to the tumor cells before irradiation with neutrons. The choice of ${ }^{10} \mathrm{~B}$ or ${ }^{157} \mathrm{Gd}$ is suggested by the high capture cross section for thermal neutrons (about 4000 barn and 255,000 barn, respectively) and by the ability of the secondary particles to release energy near the capture site. The current status of NCT has recently been reviewed ( 1 ).

To improve the efficacy of this experimental cancer treatment, various microdosimetric models have been developed

\footnotetext{
${ }^{1}$ Address for correspondence: Dipartimento di Fisica e Tecnologie Relative, Università di Palermo, Viale delle Scienze, Edificio 18, $90128 \mathrm{~Pa}-$ lermo, Italy; e-mail: marrale@difter.unipa.it.
}

to describe all the contributions to the dose inside both tumor and healthy tissues after thermal neutron irradiation (2-5). All these theoretical and/or simulation models are based on the knowledge of the properties of the neutron beams used. Therefore, many efforts have been made to characterize as accurately and precisely as possible the features of the radiation beams and their components. Many existing dosimetric techniques have been improved, and many new dosimetric systems have been discovered and developed. These techniques include thermoluminescence dosimetry (6), scintillation dosimetry through lithium gadolinium borate (7) and lithium acetate (8), chemical dosimetry with chlorobenzene-ethanol-trimethylpentane (9), and gel dosimetry (10-12).

Many studies have also been carried out with electron spin resonance (ESR), which detects the effects of ionizing radiations by measuring the concentrations of paramagnetic centers produced after exposure to radiation. The ESR technique has become an international standard for dose measurements through alanine (13). However, in the last decade various new materials have undergone detailed analyses and studies designed to improve the effectiveness of ESR in radiation detection, including various organic and inorganic compounds such as ammonium tartrate $(14,15)$, lithium lactate (16), magnesium lactate (17), and formates and dithionates (18). These compounds show good sensitivity for photon and electron beams, but the response for thermal neutrons is very inadequate. Therefore, various ways to enhance the performance of ESR dosimeters for thermal neutron beams have been examined. The most common way to increase sensitivity to thermal neutrons is the addition of nuclei with a high cross section for thermal neutron capture. However, a high capture cross section is necessary but is not sufficient for enhancing detection. The energy of the secondary particles produced after neutron capture needs to be released inside the dosimeter to be detected. These conditions are satisfied for ${ }^{6} \mathrm{Li}$, which has a cross section of about 940 barn and releases a ${ }^{3} \mathrm{H}$ ion and an $\alpha$ particle after interaction with a neutron. Recently various research groups exploited these features of ${ }^{6} \mathrm{Li}$ to improve the performance of ESR in neutron detection $(19,20)$. Another nucleus used is ${ }^{10} \mathrm{~B}$, which has a cross section of about 3840 
TABLE 1

Elemental Compositions (percentage by weight) of the $\mathrm{Gd}_{2} \mathrm{O}_{3}$-Alanine Dosimeters

\begin{tabular}{|c|c|c|c|c|c|c|c|c|}
\hline Batch & $\begin{array}{l}\qquad \mathrm{Gd}_{2} \mathrm{O}_{3} \text { mass } \\
\text { percentage inside } \\
\text { the active material }\end{array}$ & Hydrogen & Carbon & Nitrogen & Oxygen & Magnesium & Gadolinium & $\begin{array}{c}\text { Thickness }^{a} \\
(\mathrm{~mm})\end{array}$ \\
\hline I & $0 \%$ & 8.24 & 43.02 & 14.79 & 33.91 & 0.04 & 0.00 & 2.50 \\
\hline II & $3 \%$ & 8.02 & 41.89 & 14.34 & 33.27 & 0.04 & 2.44 & 2.46 \\
\hline III & $5 \%$ & 7.87 & 41.12 & 14.05 & 32.85 & 0.04 & 4.07 & 2.44 \\
\hline IV & $10 \%$ & 7.50 & 39.22 & 13.31 & 31.79 & 0.04 & 8.14 & 2.38 \\
\hline $\mathrm{V}$ & $25 \%$ & 6.39 & 33.52 & 11.09 & 28.61 & 0.04 & 20.35 & 2.20 \\
\hline VI & $50 \%$ & 4.54 & 24.02 & 7.39 & 23.31 & 0.04 & 40.70 & 1.90 \\
\hline
\end{tabular}

${ }^{a}$ Thickness of the pellets at the various gadolinium percentages.

barn and releases a ${ }^{7} \mathrm{Li}$ ion and an $\alpha$ particle after interaction with a neutron. Several studies have been carried out on the addition of boric acid enriched with ${ }^{10} \mathrm{~B}$ to alanine pellets (21-24).

In this study, we examined the enhancement of the ESR response to a mixed field (thermal neutrons and $\gamma$-ray photons) of alanine and ammonium tartrate pellets with various concentrations of gadolinium oxide. The isotopes of gadolinium that are most sensitive to thermal neutrons are ${ }^{155} \mathrm{Gd}$ and ${ }^{157} \mathrm{Gd}$, which have capture cross sections of about 75,000 and 255,000 barn, respectively. The total capture cross section of natural gadolinium is about 50,000 barn, as determined by considering the abundances of the various gadolinium isotopes. The reactions of both isotopes with thermal neutrons are very complex. Furthermore, after thermal neutron capture, these isotopes release Auger electrons and internal conversion electrons that lose their energy close to the site of capture. As an example, a simplified scheme of the reaction of ${ }^{157} \mathrm{Gd}$ with thermal neutrons is: ${ }^{157} \mathrm{Gd}+\mathrm{n}_{\mathrm{th}} \rightarrow{ }^{158} \mathrm{Gd}^{*} \rightarrow{ }^{158} \mathrm{Gd}+\gamma$-ray photons + internal conversion electrons + Auger electrons.

As mentioned above, ${ }^{157} \mathrm{Gd}$ is used as a sensitizer nucleus in NCT. The analysis of the ESR response of pellets of alanine and ammonium tartrate added with gadolinium and exposed to a mixed field (photons and thermal neutrons) is needed because, even if the probability of interaction with thermal neutrons increases with increasing gadolinium content, the use of this element inside the dosimeter produces a significant reduction or even the loss of tissue equivalence. Gadolinium has a high atomic number $\left(Z_{\mathrm{Gd}}=64\right)$, which implies a high probability of interaction with photons, especially low-energy photons, and therefore the response to a photon beam is quite different from the response of the soft tissue. The greater the gadolinium content is, the greater the differences are. Therefore, it is necessary to find the optimum balance between maximizing the sensitivity to thermal neutrons and significantly reducing tissue equivalence. In this study, we investigated how the concentration of $\mathrm{Gd}_{2} \mathrm{O}_{3}$ might enhance the neutron sensitivity of the various compounds under analysis compromising the dosimetric properties of these materials.

\section{MATERIALS AND METHODS}

Preparation of Pellets

Solid-state pellets for ESR dosimetry were made by using a mixture of L- $\alpha$-alanine (Fluka, Buchs, Switzerland) or ammonium tartrate (Carlo Erba, Italy) and gadolinium oxide $\left(\mathrm{Gd}_{2} \mathrm{O}_{3}\right.$, Aldrich Chemical Co.) in known proportions by weight.

The pellets were made by following the procedure described by Bartolotta et al. (25), which involved pressing a blend of $94 \%$ the appropriate active material $\left(\mathrm{Gd}_{2} \mathrm{O}_{3}\right.$-alanine or alanine alone, $\mathrm{Gd}_{2} \mathrm{O}_{3}$-ammonium tartrate or ammonium tartrate alone), 5\% of polyethylene (Polysciences, MW $=700$ ) as binder, and $1 \%$ of magnesium stearate (Carlo Erba, Milano, Italy) as lubricant. The mass of each pellet in this work was $28 \mathrm{mg}$. Because of the high atomic number of gadolinium $\left(Z_{\mathrm{Gd}}=64\right)$, the mass density of the pellets was increased and the thickness was reduced by the addition of gadolinium.

To investigate the enhancement of the ESR signal, we compared the ESR signals of six different batches of neutron-irradiated pellets. The batches consisted of mixtures of alanine and gadolinium oxide and mixtures of ammonium tartrate and gadolinium oxide with various mass percentages of gadolinium as described in Tables 1 and 2. The $\mathrm{Gd}_{2} \mathrm{O}_{3}$ mass percentages reported in both tables are those of gadolinium oxide inside the active material of the dosimeter. Pellets about $4 \mathrm{~mm}$ in diameter were obtained. The thicknesses of the pellets of the various blends are also given in Tables 1 and 2.

\section{Irradiations}

Irradiations were carried out inside the thermal column of the TAPIRO research nuclear reactor at the ENEA Casaccia Center near Rome. The facility used for thermalizing the fast-neutron beam produced by this reactor is HYTHOR (HYbrid Thermal spectrum sHifter TapirO Reactor), which has a beam-shaping assembly (BSA) based on a hybrid Pb-CF2RGGraphite neutron spectrum shifter configuration (26). A predominantly thermal neutron beam (about $90 \%$ of the total neutron flux) can be obtained inside the irradiation cavity with a very low $\gamma$-ray background with the HYTHOR facility. More details are given by Esposito et al. (26).

Four dosimeters were placed in suitable small cavities inside the Teflon holders prepared for neutron irradiation and irradiated inside the thermal column of the TAPIRO nuclear reactor at a thermal neutron fluence of $(18.8 \pm 0.2) \times 10^{12} \mathrm{~cm}^{-2}$ and $\gamma$-ray dose of $1.49 \pm 0.02$ Gy measured through the activation gold foils and ionization chamber, respectively.

\section{ESR Measurements}

The ESR spectra were recorded at room temperature with a Bruker ECS 106 spectrometer with a rectangular cavity $\mathrm{TE}_{102}$ operating at approximately $9.7 \mathrm{GHz}$. The peak-to-peak intensity of the central line of the spectrum was chosen as the dose-dependent quantity $(27,28)$. 
TABLE 2

Elemental Compositions (percentage by weight) of the $\mathrm{Gd}_{2} \mathrm{O}_{3}$-Ammonium Tartrate Dosimeters

\begin{tabular}{|c|c|c|c|c|c|c|c|c|}
\hline Batch & $\begin{array}{l}\qquad \mathrm{Gd}_{2} \mathrm{O}_{3} \text { mass } \\
\text { Percentage inside } \\
\text { the active material }\end{array}$ & Hydrogen & Carbon & Nitrogen & Oxygen & Magnesium & Gadolinium & $\begin{array}{c}\text { Thickness }^{a} \\
(\mathrm{~mm})\end{array}$ \\
\hline VII & $0 \%$ & 6.98 & 29.53 & 14.30 & 49.15 & 0.04 & 0.00 & 2.20 \\
\hline VIII & $3 \%$ & 6.79 & 28.79 & 13.88 & 48.06 & 0.04 & 2.44 & 2.17 \\
\hline IX & $5 \%$ & 6.67 & 28.30 & 13.59 & 47.33 & 0.04 & 4.07 & 2.15 \\
\hline X & $10 \%$ & 6.36 & 27.07 & 12.87 & 45.52 & 0.04 & 8.14 & 2.10 \\
\hline XI & $25 \%$ & 5.44 & 23.40 & 10.73 & 40.04 & 0.04 & 20.35 & 1.95 \\
\hline XII & $50 \%$ & 3.91 & 17.27 & 7.15 & 30.93 & 0.04 & 40.70 & 1.70 \\
\hline
\end{tabular}

${ }^{a}$ Thickness of the pellets at the various gadolinium percentages.

The following ESR recording parameters were chosen to obtain the highest signal-to-noise ratio for both alanine and ammonium tartrate: sweep time: $42 \mathrm{~s}$; modulation frequency: $50 \mathrm{kHz}$; number of scans accumulated: 3 .

Since the total widths of the ESR spectra of alanine and ammonium tartrate are different, the field sweeps chosen for recording the alanine and ammonium tartrate signal were $20 \mathrm{mT}$ and $10 \mathrm{mT}$, respectively. The

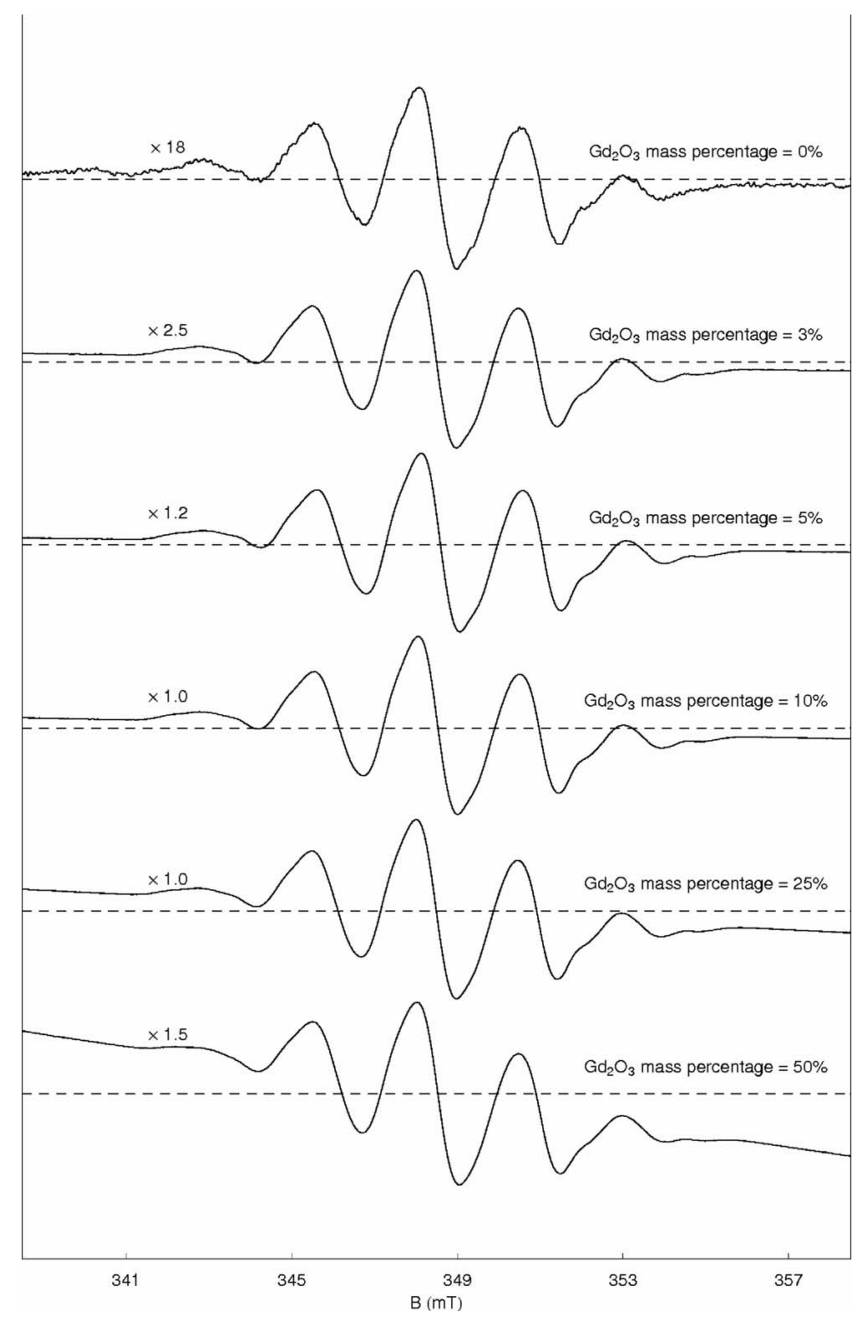

FIG. 1. ESR spectra of alanine pellets at various concentrations of gadolinium oxide exposed to the mixed field. The spectra were rescaled to have the same peak-to-peak amplitude. The factor by which each spectrum was multiplied is shown on the left side. modulation amplitude was $0.25 \mathrm{mT}$ for alanine and $0.28 \mathrm{mT}$ for ammonium tartrate. The microwave power was $6.32 \mathrm{~mW}$ for alanine and 1.59 for ammonium tartrate.

The dependence of the amplitude of the ESR signal on the orientation of each pellet inside the resonant cavity reported by Kojima et al. (29) was taken into account through averaging the ESR peak-to-peak amplitude $\left(h_{p p}\right)$ of all samples at four orientations. Then $h_{p p}$ was divided by the fractional weight content of alanine or ammonium tartrate in the sample. We used the mean value $R$ of the $h_{p p}$ values of three dosimeters with the same gadolinium content as the ESR response of the pellet for that gadolinium content. This procedure allowed for a decrease in the uncertainties associated with ESR measurements.

\section{RESULTS AND DISCUSSION}

Dosimeters of pure alanine irradiated with a mixed neutron- $\gamma$-ray field show ESR spectra similar to the wellknown spectra of alanine. Dosimeters of pure ammonium tartrate show an ESR spectrum with a single intense peak at $g \sim 2$ and two much less intense peaks on both sides of the principal peak.

In Fig. 1, the ESR spectra of $\mathrm{Gd}_{2} \mathrm{O}_{3}$-alanine pellets at various gadolinium oxide concentrations are compared. The spectra are rescaled to allow a better comparison of the line shape. Figure 2 shows the spectra of the pellets of $\mathrm{Gd}_{2} \mathrm{O}_{3}$ ammonium tartrate. The dosimeters of $\mathrm{Gd}_{2} \mathrm{O}_{3}$-alanine and $\mathrm{Gd}_{2} \mathrm{O}_{3}$-ammonium tartrate (unlike pure alanine dosimeters and pure ammonium tartrate dosimeters) also show the ESR signal due to gadolinium oxide. This signal is very wide (about $270 \mathrm{mT}$ ) and its $g$ factor is about $2(30,31)$. Therefore, the $\mathrm{Gd}_{2} \mathrm{O}_{3}$ signal in the magnetic-field range swept is linear and gives rise to the baseline shift seen in both Figs. 1 and 2. Since the intensity of this ESR signal is proportional to the amount of gadolinium inside the dosimeter, the absolute value of the slope of this linear background signal increases with increasing $\mathrm{Gd}_{2} \mathrm{O}_{3}$ concentration. To eliminate this background signal a baseline correction was performed on each spectrum of $\mathrm{Gd}_{2} \mathrm{O}_{3}$-alanine and $\mathrm{Gd}_{2} \mathrm{O}_{3}$-ammonium tartrate.

Figures 3 and 4 show the spectra for alanine and ammonium tartrate, respectively, after the baseline correction. The $\mathrm{Gd}_{2} \mathrm{O}_{3}$-alanine spectra at various concentrations shown in Fig. 3 are similar. Analogous comparisons can be made for the ESR spectra of the $\mathrm{Gd}_{2} \mathrm{O}_{3}$-ammonium tartrate do- 


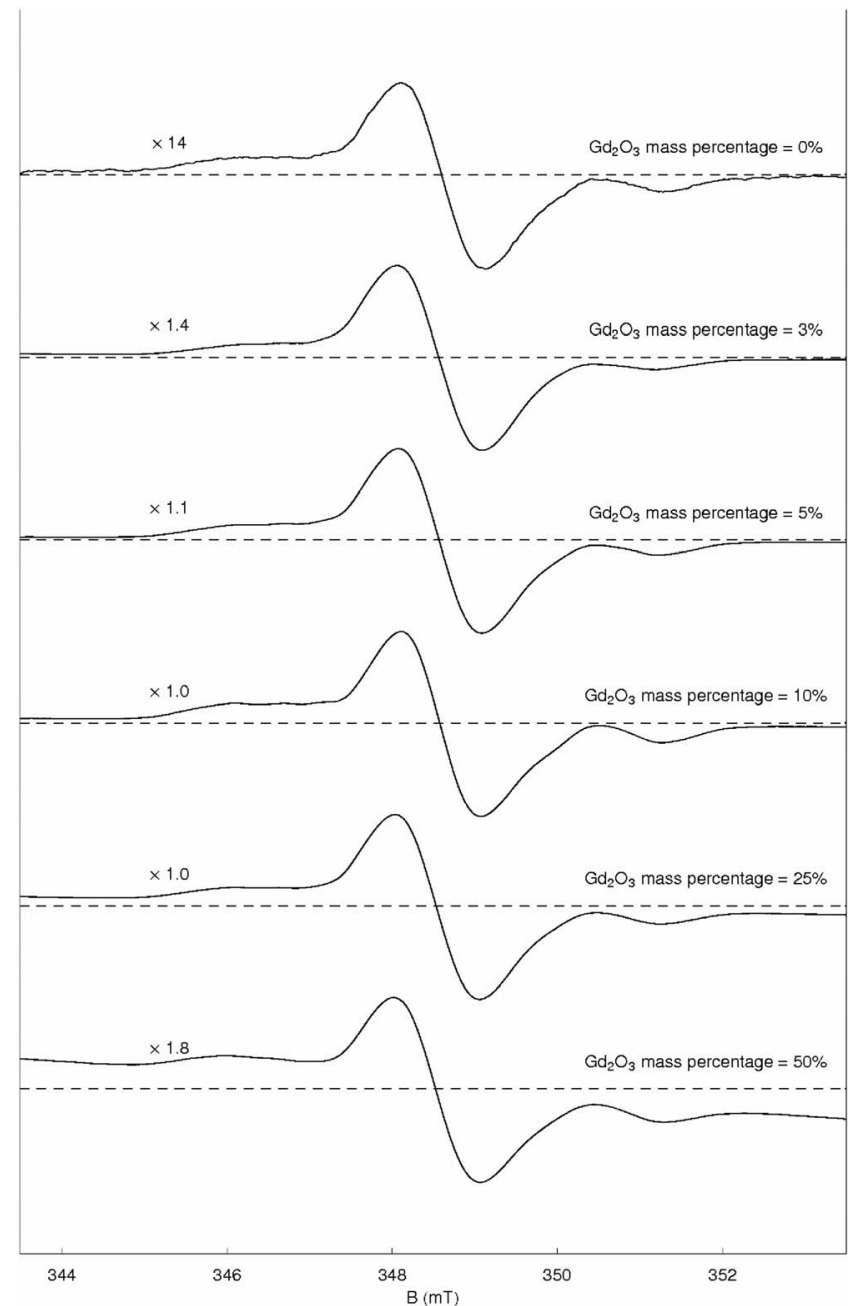

FIG. 2. ESR spectra of ammonium tartrate pellets at various concentrations of gadolinium oxide exposed to the mixed field. The spectra were rescaled to have the same peak-to-peak amplitude. The factor by which each spectrum was multiplied is shown on the left side.

simeters. The peak-to-peak amplitude $h_{p p}$ for these dosimeters was measured after this background elimination.

The ESR signal of the alanine and ammonium tartrate dosimeters exposed to the mixed field is due to both neutrons and $\gamma$-ray photons, and the ESR response to the mixed field $R_{n+\gamma}$ can be assumed to be equal to the sum of the response to neutrons $R_{n}$ and the response to $\gamma$-ray photons $R_{\gamma}$, i.e., $R_{n+\gamma}=R_{n}+R_{\gamma}$. Since we were interested in studying the ESR response due to only neutrons, we used the calibration curves for $\gamma$-ray photons obtained as described in our previous papers $(30,31)$ to remove the $\gamma$-ray contribution from the total ESR signal. In those experiments we studied the response of a dosimeter of pure alanine and $\mathrm{Gd}_{2} \mathrm{O}_{3}$-alanine $\left(50 \% \mathrm{Gd}_{2} \mathrm{O}_{3}\right.$ weight percentage) and dosimeters of pure ammonium tartrate and $\mathrm{Gd}_{2} \mathrm{O}_{3}$-ammonium tartrate $\left(50 \% \mathrm{Gd}_{2} \mathrm{O}_{3}\right.$ weight percentage), respectively, to ${ }^{60} \mathrm{Co}$ $\gamma$ rays. We found that the addition of gadolinium increased the $\gamma$-ray sensitivities by approximately a factor of 2 and decreased the lowest detectable dose. Therefore, in the pres-

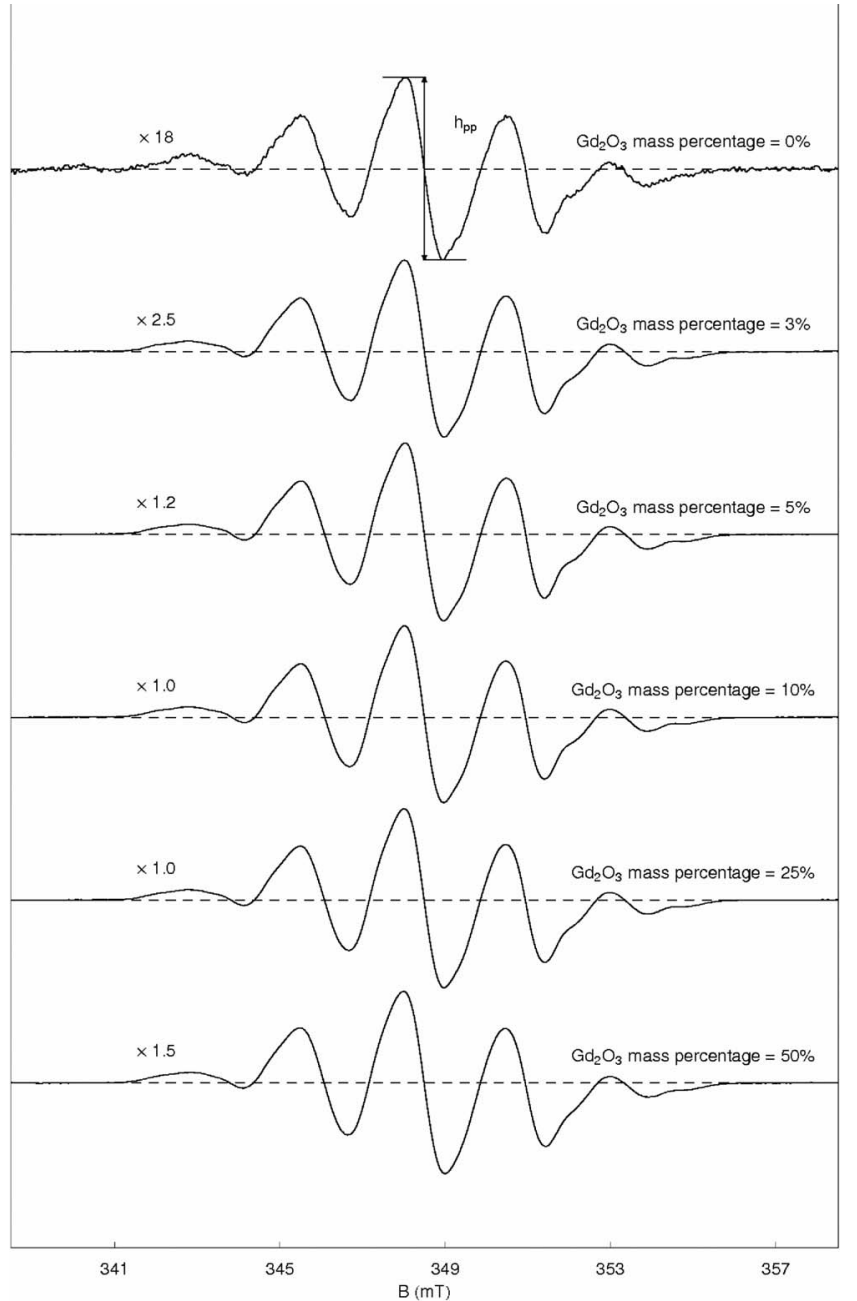

FIG. 3. ESR spectra of alanine pellets at various concentrations of gadolinium oxide after baseline correction. The peak-to-peak amplitude $h_{p p}$ is also shown.

ent work, to eliminate the $\gamma$-ray contribution to the signal in the dosimeters with alanine and ammonium tartrate with the minimum and maximum gadolinium content $(0 \%$ and $50 \%$, respectively), we estimated the ESR amplitude due to $\gamma$-ray photons $R_{\gamma}$ by using the experimental calibration functions for $\gamma$-ray photons and the $\gamma$-ray dose value provided by the irradiation center, and we subtracted it from the signal of the pellets exposed to the mixed field. The ratio $R_{\gamma} / R_{n+\gamma}$ between the $\gamma$-ray ESR signal $R_{\gamma}$ and the total ESR signal $R_{n+\gamma}$ is less than 0.08 for the dosimeters of pure alanine and pure ammonium tartrate and is less than 0.01 for dosimeters with a $50 \%$ gadolinium content.

Since we do not have a $\gamma$-ray calibration function for the dosimeters of $\mathrm{Gd}_{2} \mathrm{O}_{3}$-alanine and $\mathrm{Gd}_{2} \mathrm{O}_{3}$-ammonium tartrate with intermediate gadolinium oxide contents $(3,5,10$ and $25 \%$ ), we assumed that the $\gamma$-ray photon sensitivities and thus the ESR signal due to photons increase linearly with gadolinium content. Under this hypothesis, we estimated $R_{\gamma}$ for these gadolinium concentrations and subtracted it from the signal to get the $R_{n}$ of the response due to only the 


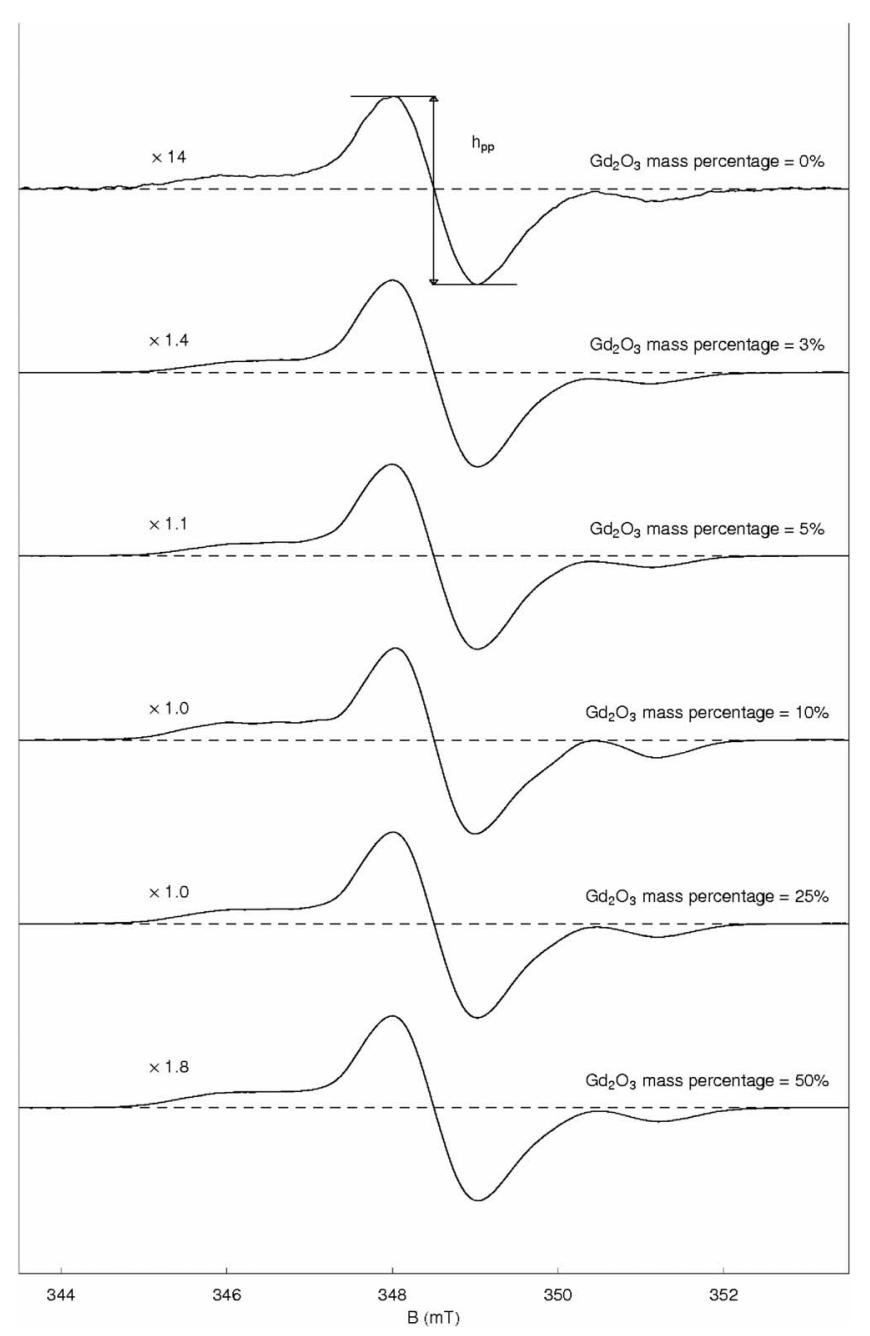

FIG. 4. ESR spectra of ammonium tartrate pellets at various concentrations of gadolinium oxide after baseline correction. The peak-to-peak amplitude $h_{p p}$ is also shown.

neutron component of the mixed field. For all these intermediate values of gadolinium content, the ratio $R_{\gamma} / R_{n+\gamma}$ is less than or equal to 0.01 . Therefore, these $R_{\gamma}$ values are comparable to or smaller than the experimental uncertainties of the $R_{n+\gamma}$ values, which are smaller than $3 \%$.

Figure 5 shows the enhancement of the ESR signal amplitude $R_{n}$ due to neutrons per alanine mass unit as a function of the gadolinium oxide percentage content for alanine dosimeters. Figure 6 shows the trend of the ESR signal for ammonium tartrate. As can be seen in both figures, $R_{n}$ for low $\mathrm{Gd}_{2} \mathrm{O}_{3}$ content is proportional to the quantity of gadolinium oxide inside the dosimeter. As the content of the additive in the mixture increases, the ESR signal reaches its maximum value and then decreases. For both the organic compounds studied, the maximum value is reached when the mass percentage of gadolinium oxide is about $25 \%$ of the active material inside the dosimeter. The signal is enhanced about 30 times for alanine dosimeters and about 20 times for ammonium tartrate dosimeters.

The trend of the ESR peak-to-peak amplitude, which in-

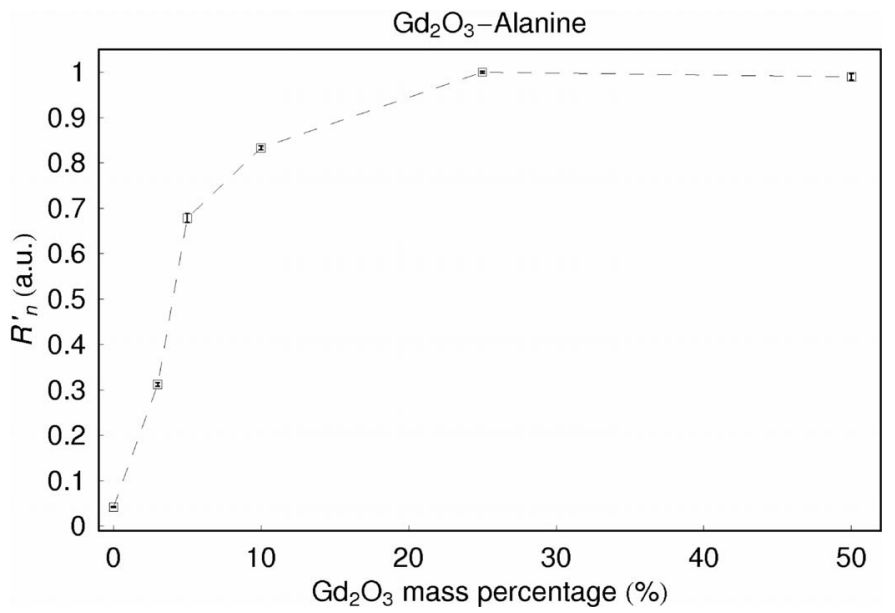

FIG. 5. Trend of the ESR signal $R^{\prime}{ }_{n}$ (equal to $R_{n}$ divided by the maximum value of the trend) due to only the neutron component of the mixed field for $\mathrm{Gd}_{2} \mathrm{O}_{3}$-alanine dosimeters. More details on the estimation of $R_{n}$ are given in the text. The error bars correspond to $\pm 1 \mathrm{SD}$.

creases with gadolinium content and reaches a maximum and then decreases, can be explained by considering all the factors that contribute to the signal and that are competing each other.

On one hand, with increasing gadolinium content, the number of targets per unit volume increases and the capture macroscopic neutron cross section, which is the capture microscopic cross section times the number of nuclei per unit volume, increases. Consequently, the capture mean free path (which is the inverse of the macroscopic neutron capture cross section) of the thermal neutrons decreases with increasing gadolinium concentration. In particular, the capture mean free path $\lambda$ of both pure organic compounds under investigation, calculated by considering the neutron reactions with hydrogen $\left(\sigma_{\mathrm{H}}^{\mathrm{C}} \sim 0.3\right.$ barn $)$ and nitrogen atoms $\left(\sigma_{\mathrm{N}}^{\mathrm{C}} \sim 1.8 \mathrm{barn}\right)$, is several hundred centimeters. For the $3 \%$ gadolinium concentration $\lambda$, calculated by considering

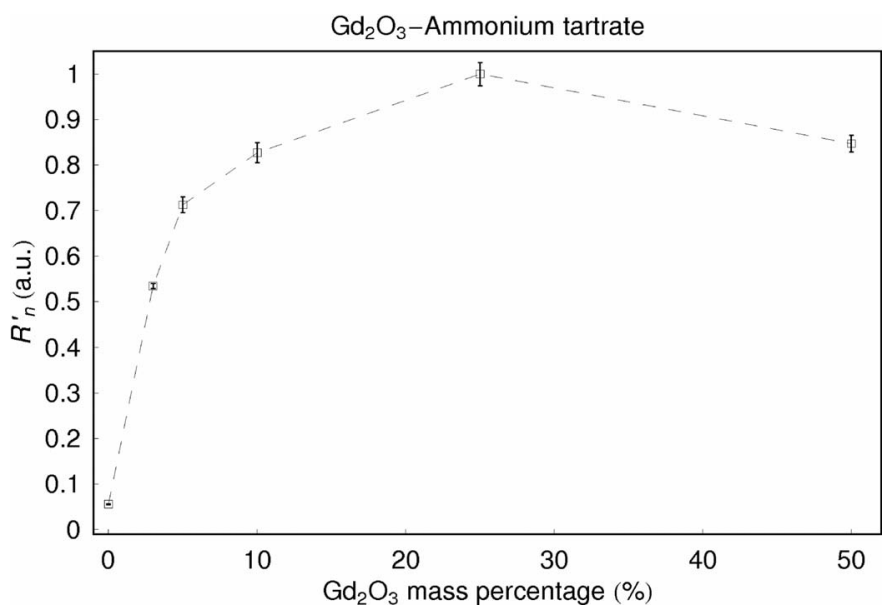

FIG. 6. Trend of the ESR signal $R^{\prime}{ }_{n}$ (equal to $R_{n}$ divided by the maximum value of the trend) due to the only neutron component of the mixed field for $\mathrm{Gd}_{2} \mathrm{O}_{3}$-ammonium tartrate dosimeters. The error bars correspond to $\pm 1 \mathrm{SD}$. 


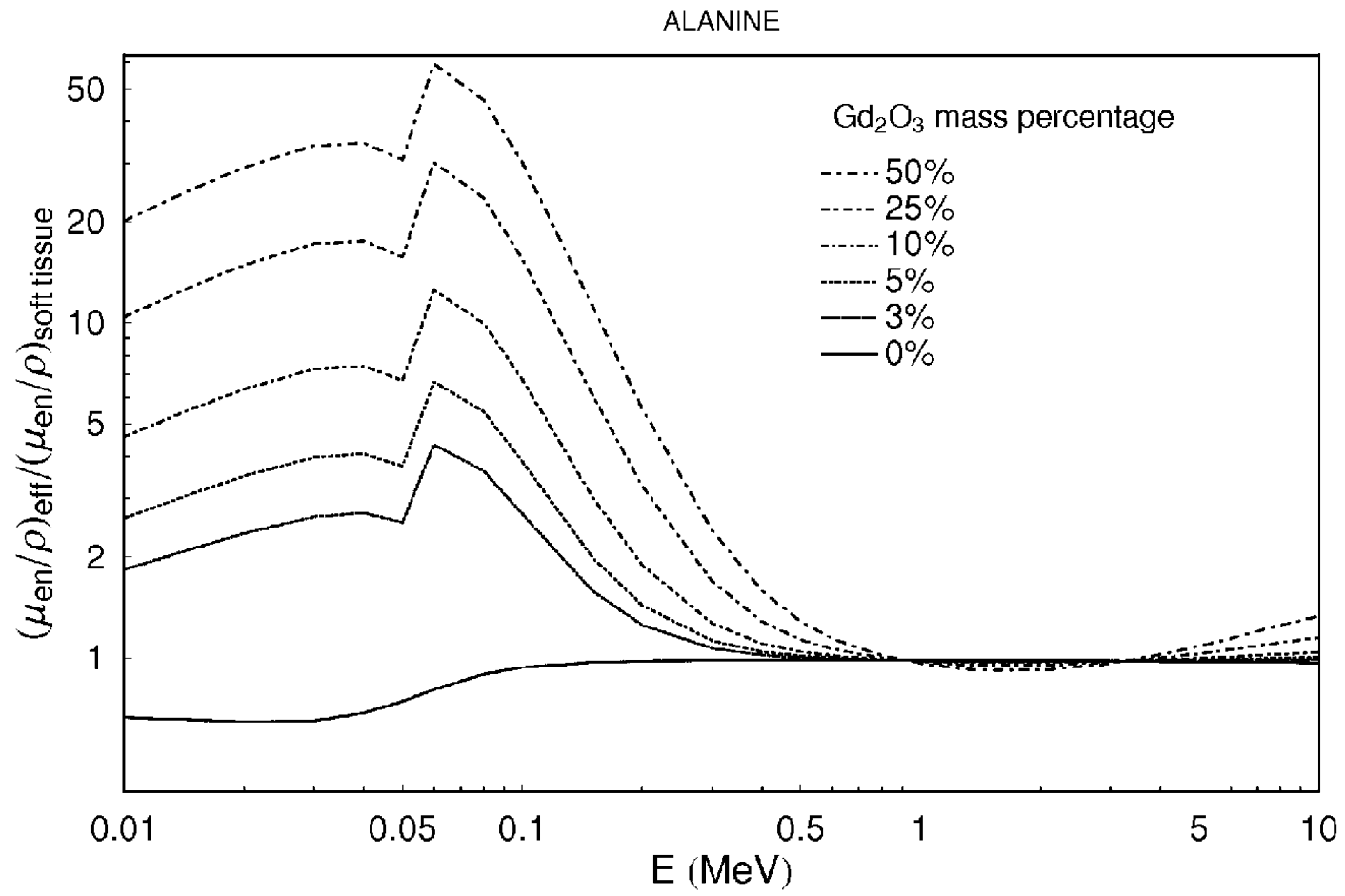

FIG. 7. The ratio of the mass energy absorption coefficient of the various mixtures constituted by alanine and gadolinium oxide to the mass energy absorption coefficient of the soft tissue as a function of the photon energy.

the reaction with gadolinium $\left(\sigma_{\mathrm{Gd}}^{\mathrm{C}} \sim 50000\right.$ barn $)$ and neglecting the other reactions, is reduced to about $2 \mathrm{~mm}$. Furthermore, increasing the amount of gadolinium inside the pellet decreases $\lambda$ up to about $0.1 \mathrm{~mm}$ for the $50 \%$ gadolinium concentration. Therefore, the probability of interaction with thermal neutrons and the number of free radicals produced by the secondary particles released after interaction of gadolinium with neutrons increase. In particular, a signal enhancement of more than 13 times for both alanine and ammonium tartrate is observed when the gadolinium mass percentage is about $5 \%$.

On the other hand, since all the dosimeters have the same mass, when the mass percentage of gadolinium oxide is increased, the amount of the sensitive compound (alanine or ammonium tartrate) inside the pellet is reduced. Therefore, even though the number of targets is large for high gadolinium concentrations, the sensitivity of the pellets decreases because the total number of molecules able to produce free radicals is reduced, and the ESR signal decreases as a consequence.

As stated previously, the aim of the present study was to maximize the ESR response in a mixed $(n, \gamma)$ radiation field. Once this response is known, it is possible to create a dosimetric system that can discriminate the neutron component from the $\gamma$-ray component $(32,33)$. Since our approach could be applied to any mixed (n, $\gamma$ ) radiation field with any neutron and photon contributions, to obtain a good estimation of the photon dose, it is necessary that the absorption and scattering properties of the pellets for photons simulate as nearly as possible those of soft tissue or other reference materials such as water, etc. Therefore, we also examined the effect of the addition of gadolinium on the tissue equivalence of the pellets. We calculated the effective mass energy absorption coefficient $\left(\mu_{e n}(E) / \rho\right)_{\text {eff }}$ of each blend as the weighted average value of the mass energy absorption coefficient $\left(\mu_{e n}(E) / \rho\right)_{\text {elem }}$ of the elements constituting the blend; i.e., $\left(\mu_{e n}(E) / \rho\right)_{\text {eff }}=\Sigma_{\text {elem }} f_{\text {elem }}\left(\mu_{e n}(E) / \rho\right)_{\text {elem }}$, where $f_{\text {elem }}$ are the mass percentages of elements. This calculation was performed for photon energies ranging from $10 \mathrm{keV}$ to $10 \mathrm{MeV}$. Then the value at energy $E$ was divided by the value of mass energy absorption coefficient $\left(\mu_{e n}(E) /\right.$ $\rho)_{\text {soft tissue }}$ of the soft tissue at the same energy $E$ reported by ICRU (34). The trends of the ratio $\left(\mu_{e n}(E) / \rho\right)_{e f f} /\left(\mu_{e n}(E) /\right.$ $\rho)_{\text {soft tissue }}$ for $\mathrm{Gd}_{2} \mathrm{O}_{3}$-alanine and $\mathrm{Gd}_{2} \mathrm{O}_{3}$-ammonium tartrate blends as a function of the photon energy are shown in Figs. 7 and 8.

For energies above $1 \mathrm{MeV}$, the ratios for the various concentrations of gadolinium oxide are quite similar to each other. Therefore, in this range of energies, the addition of the gadolinium does not significantly modify the responses of the various mixtures to the photon beam. For energies below $1 \mathrm{MeV}$, the mass energy absorption coefficient of the pellets with $50 \% \mathrm{Gd}_{2} \mathrm{O}_{3}$ become significantly greater than that of the pellets without gadolinium. Therefore, the addition of gadolinium greatly influences the response of these dosimeters to the photon beams. Another feature that must be noted is the discontinuity just above $0.05 \mathrm{MeV}$ in these trends. These abrupt increases are the $\mathrm{K}$-absorption edges of the gadolinium. They result from the fact that photoelectric absorption involving K-shell electrons cannot 


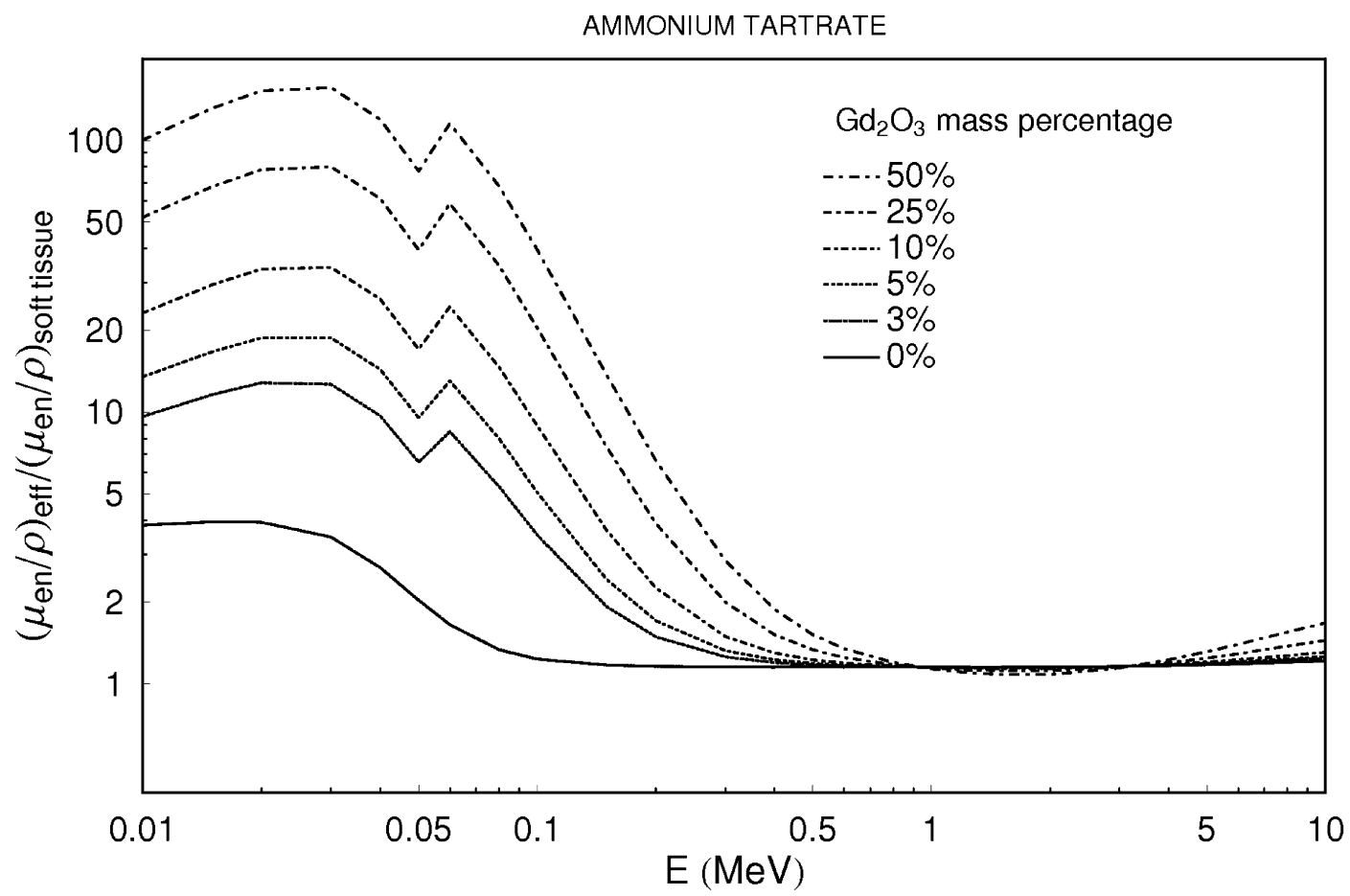

FIG. 8. The ratio of the mass energy absorption coefficient of the various mixtures constituted by ammonium tartrate and gadolinium oxide to the mass energy absorption coefficient of the soft tissue as a function of the photon energy.

occur until the photon energy exceeds the K-shell binding energy. Therefore, energies just below the edge of the photon absorption are less probable; for energies just above the edge, the photoelectric absorption is much more effective.

The differences of the trends become less evident with decreasing gadolinium concentration, as expected. For low gadolinium content (3\% and 5\%), the mass energy absorption coefficient at low energy is only a few times greater than in dosimeters without gadolinium. Consequently, for these concentrations the tissue equivalence is not excessively reduced. On the other hand, a low $\mathrm{Gd}_{2} \mathrm{O}_{3}$ content, e.g. $5 \%$, gives a 13 -fold increase in neutron sensitivity, as stated above. Therefore, to produce a reliable thermal neutron dosimeter that is also tissue equivalent, a compromise is needed.

In conclusion, the addition of gadolinium results in a promising improvement of the sensitivity for both alanine and ammonium tartrate. We studied their ESR signal after thermal neutron irradiation as a function of the gadolinium oxide concentration inside the pellets. In particular, for low gadolinium concentrations, the signal increases with the amount of gadolinium because the number of the targets and therefore the number of the secondary ionizing particles increases. However, since the total mass of the pellets in this study was kept constant, for high concentrations of gadolinium, the number of alanine or ammonium tartrate molecules from which the free radicals are formed is lower and the ESR signal decreases. Therefore, to maximize the sensitivity of the pellets a compromise between high gad- olinium oxide content and a high percentage of sensitive compound is required. Since gadolinium has a high atomic number, the addition of gadolinium involves the reduction of or even the loss of tissue equivalence. Consequently, to provide a tissue-equivalent ESR dosimeter with improved sensitivity to thermal neutrons, a low concentration of gadolinium oxide (of the order of $5 \%$ of the total mass of the dosimeter) must be chosen.

These experiments could be useful for developing thermal neutron dosimetry techniques applicable to NCT.

\section{ACKNOWLEDGMENTS}

The research described in this paper was supported by the Università di Palermo and "Dottorato in Fisica Applicata" of the Università di Palermo. The authors are grateful to Dr. Giancarlo Rosi of the ENEA Casaccia, near Rome, for the neutron irradiations and to Marcello Mirabello for technical support.

Received: June 23, 2007; accepted: September 17, 2007

\section{REFERENCES}

1. R. F. Barth, Guest Ed., Boron neutron capture therapy: A critical assessment. J. Neurooncol. 62, 1-219 (2003).

2. R. G. Zamenhof, Microdosimetry for boron neutron capture therapy: a review. J. Neurooncol. 33, 81-92 (1997).

3. C. van Vliet-Vroegindeweij, F. J. Wheeler, F. Stecher-Rasmussen, R. Moss and R. Huiskamp, Microdosimetry model for boron neutron capture therapy: I. Determination of microscopic quantities of heavy particles on a cellular scale. Radiat. Res. 155, 490-497 (2001).

4. C. van Vliet-Vroegindeweij, F. J. Wheeler, F. Stecher-Rasmussen, R. 
Moss and R. Huiskamp, Microdosimetry model for boron neutron capture therapy: II. Theoretical estimation of the effectiveness function and surviving fractions. Radiat. Res. 155, 498-502 (2001).

5. G. A. Santa Cruz and R. G. Zamenhof, The microdosimetry of the ${ }^{10} \mathrm{~B}$ reaction in boron neutron capture therapy: A new generalized theory. Radiat. Res. 162, 702-710 (2004).

6. M. Toivonen, V. Chernov, H. Jungner, C. Aschan and A. Toivonen, The abilities of LiF thermoluminescent detectors for dosimetry at boron neutron capture therapy beams. Radiat. Meas. 29, 373-377 (1998).

7. J. B. Czirr, D. B. Merrill, D. Buehler, T. K. McKnight, J. L. Carroll, T. Abbott and E. Wilcox, Capture-gated neutron spectrometry. Nucl. Instrum. Methods A 476, 309-312 (2002).

8. L. J. Rakovan, T. E. Blue and A. L. Vest, Neutron dosimetry in boron neutron capture therapy using aqueous solutions of lithium acetate. Nucl. Instrum. Methods A 414, 357-364 (1998).

9. S. Miljanić, Đ. Miljanić, E. Krištof and R. Ilić, Towards a chemical dosimetry system for boron neutron capture therapy. Nucl. Instrum. Methods A 354, 595-597 (1995).

10. G. Gambarini, S. Agosteo, P. Marchesi, E. Nava, P. Palazzi, A. Pecci, G. Rosi and R. Tinti, Discrimination of various contributions to the absorbed dose in BNCT: Fricke-gel imaging and intercomparison with other experimental results. Appl. Radiat. Isot. 53, 765-772 (2000).

11. G. Gambarini, C. Birattari, C. Colombi, L. Pirola and G. Rosi, Frickegel dosimetry in boron neutron capture therapy. Radiat. Prot. Dosimetry 101, 419-422 (2002).

12. G. Gambarini, V. Colli, S. Gay, C. Petrovich, L. Pirola and G. Rosi, In-phantom imaging of all dose components in boron neutron capture therapy by means of gel dosimeters. Appl. Radiat. Isot. 61, 759-763 (2004).

13. K. Mehta and R. Girzikowsky, IAEA reference dosimeter: alanineESR. In Techniques for High Dose Dosimetry in Industry, Agriculture and Medicine-Proceedings of the Symposium, pp. 299-304. IAEA-TECDOC-1070, IAEA, Vienna, 1999.

14. S. Olsson, S. Bagherian, E. Lund, G. A. Carlsson and A. Lund, Ammonium tartrate as an ESR dosimeter material. Appl. Radiat. Isot. 50, 955-965 (1999).

15. A. Bartolotta, M. C. D’Oca, M. Brai, V. Caputo, V. De Caro and L. I. Giannola, Response characterization of ammonium tartrate solid state pellets for ESR dosimetry with radiotherapeutic photon and electron beams. Phys. Med. Biol. 46, 461-471 (2001).

16. G. M. Hassan, M. Ikeya and S. Toyoda, Lithium lactate as an ESR dosimeter Appl. Radiat. Isot. 49, 823-828 (1998).

17. G. M. Hassan, M. Ikeya and S. Takaki, Radiation-induced defects in magnesium lactate as ESR dosimeter Radiat. Meas. 30, 189-196 (1999).

18. E. Lund, H. Gustafsson, M. Danilczuk, M. D. Sastry, A. Lund, T. A. Vestad, E. Malinen, E. O. Hole and E. Sagstuen, Formates and dithionates: sensitive EPR-dosimeter materials for radiation therapy Appl. Radiat. Isot. 62, 317-324 (2005).
19. E. Lund, H. Gustafsson, M. Danilczuk, M. D. Sastry and A. Lund, Compounds of ${ }^{6} \mathrm{Li}$ and natural $\mathrm{Li}$ for EPR dosimetry in photon/neutron mixed radiation fields. Spectrochim. Acta A 60, 1319-1326 (2004).

20. E. Herrera, F. Ureña-Nuñez and A. Delfín Loya, Lithium carbonate $\left(\mathrm{Li}_{2} \mathrm{CO}_{2}\right)$ as a material for thermal neutron fluence measurements Appl. Radiat. Isot. 63, 241-246 (2005).

21. S. Galindo and F. Ureña-Nuñez, EPR signal enhancement of alanine irradiated with thermal neutrons Radiat. Res. 133, 387-389 (1993).

22. F. Ureña-Nuñez, S. Galindo and J. Azorin, An alanine-boron compound for thermal neutron fluence measurements. Part 1: Synthesis and development. Appl. Radiat. Isot. 49, 1657-1664 (1998).

23. F. Ureña-Nuñez, S. Galindo and J. Azorin, An alanine-boron compound for thermal neutron fluence measurements. Part 2: EPR response. Appl. Radiat. Isot. 50, 763-767 (1999).

24. A. Bartolotta, M. C. D’Oca, B. Lo Giudice, M. Brai, R. Borio, N. Forini, P. Salvatori and S. Manera, Combined TL and ${ }^{10} \mathrm{~B}$-alanine ESR dosimetry for BNCT Radiat. Prot. Dosimetry 110, 627-630 (2004).

25. A. Bartolotta, M. Brai, V. Caputo, V. De Caro, L. I. Giannola and G. Teri, ESR solid state dosimetry: behaviour of various amino acids and blend preparation procedures. Radiat. Prot. Dosimetry 84, 293 296 (1999).

26. J. Esposito, G. Rosi and S. Agosteo, The new hybrid thermal neutron facility at TAPIRO reactor for BNCT radiobiological experiments Radiat. Prot. Dosimetry, in press. [doi:10.1093/rpd/ncm015]

27. D. F. Regulla and U. Deffner, Dosimetry by ESR spectroscopy of alanine. Int. J. Appl. Radiat. Isot. 33, 1101-1114 (1982).

28. American Society for Testing and Materials, Standard Practice for Use of the Alanine-EPR Dosimetry System. ASTM E1670-94, ASTM, Philadelphia, 1995.

29. T. Kojima, S. Kashiwazaki, H. Tachibana, R. Tanaka, M. F. Desrosiers and W. L. McLaughlin, Orientation effects on ESR analysis of alanine-polymer dosimeters. Appl. Radiat. Isot. 46, 1407-1411 (1995).

30. M. Brai, G. Gennaro, M. Marrale, A. Bartolotta and M. C. D'Oca, ESR response to $\gamma$-rays of alanine pellets using $\mathrm{B}(\mathrm{OH})_{3}$ or $\mathrm{Gd}_{2} \mathrm{O}_{3}$. Appl. Radiat. Isot. 65, 435-439 (2007).

31. M. Brai, G. Gennaro, M. Marrale, L. Tranchina, A. Bartolotta and M. C. D'Oca, ESR response to ${ }^{60} \mathrm{Co} \gamma$-rays of ammonium tartrate pellets using $\mathrm{Gd}_{2} \mathrm{O}_{3}$ as additive. Radiat. Meas. 42, 225-231 (2007).

32. ICRU, Neutron Dosimetry for Biology and Medicine. Report 26, International Commission on Radiation Units and Measurements, Bethesda, MD, 1977.

33. M. Brai, M. Marrale, G. Gennaro, A. Bartolotta, M. C. D'Oca and G. Rosi, Improvement of ESR dosimetry for thermal neutron beams through gadolinium addition. Phys. Med. Biol., 52, 5219-5230 (2007)

34. ICRU, Tissue Substitutes in Radiation Dosimetry and Measurements. Report 44, International Commission on Radiation Units and Measurements, Bethesda, MD, 1989 